\title{
A 22-Year-Old College Athlete With Abdominal Symptoms
}

Ronald N. Rubin, $\mathrm{MD}^{1,2}$-Series Editor

A 22-year-old woman presents to urgent care with a 2-day history of progressive abdominal discomfort. She first noticed the vague onset of generalized abdominal discomfort and anorexia. This was not relieved by bowel movements. Over the next 2 days, the discomfort lateralized to the right lower quadrant and has progressively worsened. She did not report any emesis.

She is otherwise quite healthy with no chronic major medical diagnoses. She has used oral contraceptives for several years without difficulty. Her menses are light but normal and are not accompanied by cramps. Her last menstrual period was 2 weeks ago.

She attends university and is a scholarship varsity basketball athlete. Basketball is in the off season, but she has a program to maintain cardiovascular and muscular fitness until the formal preseason training program begins 3 months from now, with the regular season starting thereafter.

Physical examination findings showed a well-developed woman most comfort- able in a flexed hip position. She has a temperature of $37.5^{\circ} \mathrm{C}$, a pulse rate of 92 beats/min, and a blood pressure of $110 / 70$ $\mathrm{mm} \mathrm{Hg}$. Pertinent findings include $3+$ tenderness to direct palpation in the right lower quadrant with a component of rebound. Rectal examination findings were negative, including results of a hematest of her stool.

Results of a laboratory screening showed normal levels of hemoglobin and platelets with a white blood cell count of $10,800 / \mathrm{mm}^{3}$, with $88 \%$ polymorphonuclear leukocyte forms and $4 \%$ band forms. $A$ urinalysis was negative for red and white blood cells. A discussion of management was initiated pending a radiologic study.

Which of the following is the most accurate statement regarding the management of this patient?

A. Little helpful additional data will be forthcoming from radiologic testing, and discharge on antibiotics is optimal.

B. A computed tomography (CT) scan confirming or refuting the presence of

\section{AFFILIATIONS:}

'Lewis Katz School of Medicine at Temple University, Philadelphia, Pennsylvania

${ }^{2}$ Department of Medicine, Temple University Hospital, Philadelphia, Pennsylvania

\section{CITATION:}

Rubin RN. A 22-year-old college athlete with abdominal symptoms. Consultant. 2022;62(2):e36-e38. doi:10.25270/con.2022.01.00006

\section{DISCLOSURES:}

The author reports no relevant financial relationships.

\section{CORRESPONDENCE:}

Ronald N. Rubin, MD, Temple University Hospital, 3401 N Broad Street, Philadelphia, PA 19140 (blooddocrnr@yahoo.com)

appendicoliths is an absolute contraindication to antibiotics as the choice of therapy.

C. Overall results are essentially equivalent for surgery or antibiotics in this situation, but about $30 \%$ of patients receiving antibiotics will need appendectomy after 3 to 12 months.

D. Total adverse effects of therapy are strikingly different comparing both treatment strategies, antibiotics, or surgery.

\section{Correct Answer: C}

I should hope that all of you quickly recognized that the clinical entity being described in the patient vignette was acute appendicitis, still among the most common abdominal indications for surgery in the United States. As will be further discussed below, there is an ongoing data-generated competition in regard to the superb technological advances in diagnosing the condition and accurately defining its extent and pathology (eg, perforated or not, appendicolith or not, and the actual presence or not of acute appendicitis in the first place) by means of ever more accurate and viable abdominal CT and ultrasound techniques. Additionally, the advent of laparoscopic appendectomy in the vast majority of the United States has made the procedure safer and more complication-free than ever. This brings us to a discussion of the data surrounding the question of how to best manage acute appendicitis, early (even immediate) surgery vs a course of antibiotics with follow-up.

But first, we should discuss the diagnosis of acute appendicitis. There is a great body of validated data using the Alvarado 
Score to make a clinical diagnosis of appendicitis with great accuracy. The Alvarado Score uses point assignments for a number of easily obtainable clinical findings-migration of abdominal pain (1 point), anorexia (1 point), nausea (1 point), right lower quadrant tenderness (2 points), rebound pain (1 point), elevated temperature above $37{ }^{\circ} \mathrm{C}$ (1 point), leukocytosis ( 2 points), and white blood cell left shift (1 point). A point score of 1 to 4 indicates appendicitis is unlikely, 5 to 6 is indeterminate, and 7 to 10 means surgery is indicated. 'Our patient's Alvarado Score was 9 and, even 30 years ago, would have required surgery with no additional testing. In the time interval since, however, there has been improvement and ease of ultrasound and CT techniques that can confirm or refute acute appendicitis in essentially $100 \%$ of cases and demonstrate other details of the situation such as perforation and the presence of appendicolith. For now, let's just accept the strong Alvarado Score as evidence for the presence of acute appendicitis in the presented patient and proceed to the meat of the article-management of acute appendicitis.

In the present era, the dominant question is, "What is the optimal management of acute appendicitis: immediate surgery or antibiotics?" In the last several years, a series of excellent studies have shown what can be expected in the short and long term for both these strategies. The very good news is that almost all patients will do well. At issue is how they get there and what seems optimal for any specific patient.

The first strong trial, the so-called APPAC randomized clinical trial, compared immediate surgery (273 patients) to antibiotics (257 patients) with a very strong 5-year follow-up. ${ }^{2}$ The researchers found that $27.3 \%$ of patients receiving antibiotics required subsequent appendectomy after 1 year and a cumulative need for subsequent appendectomy by year 5 of $39 \%$. Most recurrent episodes requiring appendectomy occurred within the first 2 years. The antibiotic group were saved an

\section{WHAT'S THE TAKE HOME?}

Appendectomy remains the most common cause and indication for abdominal surgery in the United States. There has been a degree of controversy in the recent literature as to the issue of immediate surgery vs therapy with antibiotics. Several large studies have provided data comparing these 2 strategies. The best 2 are the APPAC trial ${ }^{2}$ and the more recent CODA collaborative trial. ${ }^{3}$ Both demonstrated "noninferiority" in the antibiotic group, with eventual appendectomy being required within 90 to 360 days in about $30 \%$ of patients initially treated with antibiotics. There were differences in the groups regarding measurements such as length of hospital stay (which was quite short in both groups), missed workdays, and other rather soft end points (in the author's opinion). ${ }^{2}$ The newer CODA study compared laparoscopic surgery rather than an open appendectomy, which very much improved many of the downstream differences between antibiotics vs surgery. The conclusions were that the choice depends on patient preference and life priorities rather than

$8.8 \%(n=24)$ infection complication rate, mainly incisional, and a $1.5 \%(n=4)$ incisional hernia rate compared with the surgery group. It must be stressed that more than $90 \%$ of the patients undergoing surgery received an open appendectomy in this trial, which is likely not the surgical standard in the United States now. The researchers' appropriate conclusion was that antibiotics are a "feasible" treatment in acute appendicitis. ${ }^{2}$

A newer and stronger randomized trial-the CODA collaborative trial-again compared antibiotics/follow-up with appendectomy but with even more patients in each group $(n=776)$ and the added features of actual radiological diagnosis confirmation by either CT and/or ultrasound in essentially $100 \%$ of patients such that pathologic features including presence of appendicolith and appendiceal perforation could be determined. ${ }^{3}$ And, very importantly, almost all (96\%) of the surgical group had laparoscopic surgery vs open appendectomy. The findings again showed that overall prognosis was outstanding; no deaths were reported. In the antibiotic group, $11 \%$ of patients had experienced a subsequent need for appendectomy at 48 hours, $20 \%$ at 30 days, and $29 \%$ at 90 days with the curve flattening thereafter. There seemed to be a higher incidence of subsequent need for surgery when an appendicolith was present on imaging ( $41 \%$ at 90 days). ${ }^{3}$ The cost of adverse events occurring in the early appendectomy group was a low $2.7 \%(n=21)$ wound infection rate, far less than the APPAC trial and surely caused by the superior laparoscopic procedure used in CODA. ${ }^{2,3}$ You may note that I used the word "definitive" for the laparoscopic surgery described in the CODA paper. Significant verbiage was spent on softer end points including workdays missed, and actual time in hospital (hours rather than days) was no different. I show my age when I dismiss these softer end points. I will let politics, economists, MD/MPH personnel, and chief medical officers deal with these while I remain an advocate for the morbidity and mortality benefit of the patient directly in front of me.

Yes, there is now strong data that with proper clinical criteria and radiologic corroboration that most acute appendicitis cases have 2 alternatives to management: immediate surgery or antibiotics with follow-up. With antibiotics 
about one-third of patients will require an appendectomy within 1 to 2 years of follow-up, so two-thirds will not undergo a surgical procedure. A positive note it may seem, but the mean age in the trials discussed was 34 years in the APPAC trial $^{2}$ and 38 years in the CODA trial ${ }^{3}$, both age groups with very low risk for surgery/ anesthesia. Meanwhile the surgery group has had their pathology resected definitively at the small cost (in my opinion) of a $2.7 \%$ risk for wound infection and a bit more time of upfront work missed.

As commented in an accompanying editorial, it would seem that most health care providers would offer laparoscopic surgery and most patients would choose laparoscopic surgery when offered. ${ }^{4}$ The clinical vignette was constructed with a significant timing issue in the history, which makes the decision even more clear cut (and Answer C the correct one). The patient has bona-fide and important timelines she must navigate. She is a scholarship student with a sports schedule that makes getting her appendicitis definitively resolved now and without risk for further ambush events months down the line the optimal strategy. Answer $A$ is incorrect. Radiology can add to anatomy and pathology information, particularly whether or not an appendicolith is present, which seems to increase the failure rate of an antibiotic regimen. Having said that, appendicoliths are not an absolute contradiction to an antibiotic regimen, as a full $60 \%$ of such cases will do well despite its presence, making Answer B incorrect as well.

\section{Patient Follow-Up}

CT scanning confirmed the presence of acute appendicitis. The available data was discussed in detail with the patient and her family. Her schedule regarding her athletic participation had a roughly 3-month "gap" before practices and intensive physical training were to begin. She wanted to avoid any recurrence and need for future surgery that might encroach on that schedule and very much preferred getting this issue definitively resolved. She chose to undergo laparoscopic appendectomy, which was performed the following day. There were no complications, and she was discharged within 24 hours. She resumed normal activities by 30 days and is on schedule to resume her varsity basketball program going forward.

\section{What's the Take Home?}

Appendectomy remains the most common cause and indication for abdominal surgery in the United States. There has been a degree of controversy in the recent literature as to the issue of immediate surgery vs therapy with antibiotics. Several large studies have provided data comparing these 2 strategies. The best 2 are the APPAC trial $^{2}$ and the more recent CODA collaborative trial. ${ }^{3}$ Both demonstrated "noninferiority" in the antibiotic group, with eventual appendectomy being required within 90 to 360 days in about $30 \%$ of patients initially treated with antibiotics. There were differences in the groups regarding measurements such as length of hospital stay (which was quite short in both groups), missed workdays, and other rather soft end points (in the author's opinion). ${ }^{2}$ The newer CODA study compared laparoscopic surgery rather than an open appendectomy, which very much improved many of the downstream differences between antibiotics vs surgery. The conclusions were that the choice depends on patient preference and life priorities rather than bona-fide medical differences in outcome, a conclusion with which it is always difficult to argue. Still, the option of a quick, safe, and effective procedure that resolves the pathology definitively, a veritable "holy grail" for any surgeon, seems even more difficult to refute.

\section{References}

1. Alvarado A. A practical score for the early diagnosis of acute appendicitis. Ann Emerg Med. 1986;15(5):557-564. https://doi. org/10.1016/s0196-0644(86)80993-3

2. Salminen $P$, Paajanen $H$, Rautio $T$, et al. Antibiotic therapy vs appendectomy for treatment of uncomplicated acute appendicitis: the APPAC randomized clinical trial. JAMA, 2015;313(23):2340-2348. https://doi org/10.1001/jama.2015.6154

3. CODA Collaborative, Flum DR, Davidson $\mathrm{GH}$, et al. A randomized trial comparing antibiotics with appendectomy for appendicitis. N Engl J Med. 2020;383(20):1907-1919. https://doi.org/10.1056/nejmoa2014320

4. Jacobs D. Antibiotics for appendicitis - proceed with caution. N Engl J Med 2020;383(20):1985-1986. https://doi. org/10.1056/nejme2029126 